Review Article

\title{
High-throughput Imaging: Focusing In On Drug Discovery in 3D
}

\author{
Linfeng Li ${ }^{\mathrm{a}}$, Qiong Zhou ${ }^{\mathrm{a}}$, Ty C. Voss ${ }^{\mathrm{c}}$, Kevin L. Quick ${ }^{\mathrm{c}}$, and Daniel V. LaBarbera ${ }^{\mathrm{a}, \mathrm{b}, *}$ \\ ${ }^{a}$ Department of Pharmaceutical Sciences, Skaggs School of Pharmacy and \\ Pharmaceutical Sciences, University of Colorado Anschutz Medical Campus, Aurora, CO \\ 80045, United States \\ ${ }^{\mathrm{b}}$ The University of Colorado Cancer Center, Developmental Therapeutics Program, \\ University of Colorado Anschutz Medical Campus, Aurora, CO 80045, United States \\ ${ }^{c}$ PerkinElmer Inc., 940 Winter Street, Waltham, Massachusetts, 02451, United States
}

*Corresponding author: Daniel V. LaBarbera, Department of Pharmaceutical Sciences, Skaggs School of Pharmacy and Pharmaceutical Sciences, University of Colorado Anschutz Medical Campus, Aurora, CO 80045

E-mail address: Daniel.LaBarbera@ucdenver.edu

C 2015. This manuscript version is made available under the Elsevier user license http://www.elsevier.com/open-access/userlicense/1.0/ 


\begin{abstract}
3D organotypic culture models such as organoids and multicellular tumor spheroids (MCTS) are becoming more widely used for drug discovery and toxicology screening. As a result, 3D culture technologies adapted for high-throughput screening formats are prevalent. While a multitude of assays have been reported and validated for highthroughput imaging (HTI) and high-content screening (HCS) for novel drug discovery and toxicology, limited HTI/HCS with large compound libraries have been reported. Nonetheless, 3D HTI instrumentation technology is advancing and this technology is now on the verge of allowing for 3D HCS of thousands of samples. This review focuses on the state-of-the-art high-throughput imaging systems, including hardware and software, and recent literature examples of 3D organotypic culture models employing this technology for drug discovery and toxicology screening.
\end{abstract}

Key words: 3D tissue culture; High-throughput imaging; High-content screening; Multicellular tumor spheroids; Organoids; 3D Image analysis software 


\section{Introduction}

Multicellular organisms emerged on Earth over 600 million years ago. Of these organisms, marine sponges are recognized as the oldest surviving animal lineage [1]. Evolutionarily, the origins of cell polarity, adhesion, and epithelial structures first appear in sponges. Therefore, it is fitting that in 1907, H.V. Wilson reported that isolated cells from silicious sponges, aggregated in masses, could self assemble to regenerate a perfect sponge [2]. This seminal observation represents one of the first organotypic three dimensional (3D) tissue culture methods conducted in a laboratory setting [3]. Interestingly, Wilson described the sponge cell masses as forming a "spheroid" structure, which has proved to be a common feature and descriptor of many organotypic culture models, termed organoids. Over the past 100 years, 3D organoids have revolutionized our understanding of human development and disease, including pluripotent and embryonic stem cells and the development of organoid models of the breast, gut, brain, kidney, retina, and other organs $[3,4]$. Organoids are defined by their ability to mimic aspects of organ function and have key features such as multiple cell types, including stem and progenitor cells, and the distinct ability to self assemble [3].

Like organoids, tumoroids or multicellular tumor spheroids (MCTS) [5], mimic in vivo tumor biology and aspects of the tumor microenvironment (e.g. extracellular matrix, ECM) [6]. Also like organoids, MCTS can self assemble, contain more than one cell type, and harbor subpopulations of cancer stem cells (CSC) [7, 8]. MCTS display in vivo tumor function with zones of proliferation, quiescence and hypoxia, and gradients of oxygen and nutrients $[9,10]$. Moreover, MCTS mimic in vivo human tumor gene expression better compared to monolayer cell culture [11-14]. Over the past ten years there has been 
a paradigm shift in high-throughput screening (HTS) placing 3D phenotypic tissue culture models at the forefront of drug discovery $[4,15,16]$. In particular, the MCTS model has proved to be ideal for cancer drug discovery and thus has been adapted for use with numerous HTS technologies, including microfluidics, micropatterning, and automated robotic liquid handling and detection [6]. This review will focus on 3D phenotypic high-throughput imaging (HTI) systems, including hardware and software, and recent work towards achieving HTI and multiparametric high-content screening (HCS) for drug discovery using organoids and MCTS.

\section{3D HTI technology and analysis software}

\subsection{Hardware}

3D HTI requires techniques that are able to overcome and penetrate tissue depths and their associated light scattering, allowing for quantitative analysis of cellular phenotypes in 3D culture models such as organoids and MCTS. A dynamic quantitative analysis of such biological structures requires an excellent signal-to-noise ratio, optical sectioning capability, large field of view, good spatial resolution, a fast image stack recording rate, and a low sample fluorophore excitation level [17]. Confocal fluorescence microscopy represents the state-of-the-art imaging technology to reveal the localization and intensity

of fluorescent reporters. In confocal imaging, optical sectioning is achieved by the combination of point illumination and point detection, eliminating out-of-focus light from contributing to the exposure. Recent advancements in HCS technology are enabling confocal-based imaging and analysis of complex 3D organoids and MCTS (Table 1). 
PerkinElmer's Operetta ${ }^{\mathrm{TM}}$ and the new Opera Phenix ${ }^{\mathrm{TM}}$ high-content imaging systems utilize a confocal spinning-disk design, which provides high-resolution images of 3D cell-culture models. The Opera Phenix ${ }^{\mathrm{TM}}$ has an advanced spinning-disk technology called Synchrony ${ }^{\mathrm{TM}}$ Optics, giving significantly improved 3D image resolution and throughput. Synchrony ${ }^{\mathrm{TM}}$ Optics reduces spectral cross talk by placing excitation lasers into two groups such that spectrally neighboring laser lines are in different lightpaths, allowing for up to four channels of simultaneous imaging (Supplementary Fig. S1). These two lightpath groups pass through two different locations on the spinning micro-lens pinhole disk so that spectrally adjacent emission is blocked by the pinhole patterns. Additionally, the spinning disk on the Opera Phenix ${ }^{\mathrm{TM}}$ utilizes an increased pinhole to pinhole distance to reduce spatial cross talk, which is critical for imaging 3D samples. In addition to these technologies, scientific complementary metal-oxide semiconductor (sCMOS) camera and a high numerical aperture water immersion objectives allow for high sensitivity, resolution and speed required for 3D imaging.

GE Healthcare's IN Cell Analyzer 6000 is a laser-based line scanning confocal imaging platform, it features an optical system that incorporates an iris-like variable aperture design (Supplementary Fig. S2) that is different from conventional point scanning confocal microscopy. IN Cell Analyzer 6000 uses a large field-of-view sCMOS camera to image samples line-by-line and the camera's electronic rolling shutter as a virtual confocal slit to block the out of focus light. This unique design facilitates removal of the unwanted fluorescent background without significant loss of sample signal and controls the degree of confocality on each fluorescent channel, resulting in optimized confocal image quality. The highly sensitive sCMOS detector chip along with laser-based 
illumination provides IN Cell Analyzer 6000 with tremendous sensitivity for 3D imaging that is comparable to the laser-based spinning disk (Nipkow) technology.

Table 1. Available 3D high-throughput imaging and high-content imaging systems.

\begin{tabular}{|c|c|c|}
\hline 3D HTI Systems & Company & Key Technology \\
\hline Opera Phenix & PerkinElmer & Confocal (laser based) \\
\hline Operetta & PerkinElmer & Confocal (lamp based) \\
\hline IN Cell Analyzer 6000 & GE Healthcare & Confocal (laser based) \\
\hline ImageXpress Ultra & $\begin{array}{l}\text { Molecular } \\
\text { Devices }\end{array}$ & Confocal (laser based) \\
\hline $\begin{array}{l}\text { ImageXpress Micro } \\
\text { Confocal }\end{array}$ & $\begin{array}{l}\text { Molecular } \\
\text { Devices }\end{array}$ & Confocal (LED based) \\
\hline Arrayscan XTI & ThermoFisher & Confocal (LED based) \\
\hline CellInsight CX7 & ThermoFisher & Confocal (LED based) \\
\hline Cell Voyager 7000S & Yokogawa & Confocal (laser based) \\
\hline CQ1 & Yokogawa & Confocal (laser based) \\
\hline Acumen Cellista & TTP LabTech & $\begin{array}{l}\text { Laser-scanning } \\
\text { fluorescence }\end{array}$ \\
\hline ImageXpress Velos & $\begin{array}{l}\text { Molecular } \\
\text { Devices }\end{array}$ & $\begin{array}{l}\text { Laser-scanning } \\
\text { fluorescence }\end{array}$ \\
\hline $\begin{array}{l}\text { ImageXpress Micro } \\
\text { XLS }\end{array}$ & $\begin{array}{l}\text { Molecular } \\
\text { Devices }\end{array}$ & Phase contrast/widefield \\
\hline Celigo & Nexcelom & Brightfield \\
\hline Cell ${ }^{3}$ iMager & InSphero & Brightfield \\
\hline 3D Imaging Systems & Company & Key Technology \\
\hline A1 MP+/ A1R MP+ & Nikon & Multiphoton/confocal \\
\hline Lightsheet Z.1 & ZEISS & Light-sheet fluorescence \\
\hline IncuCyte ZOOM & $\begin{array}{l}\text { Essen } \\
\text { Bioscience }\end{array}$ & $\begin{array}{l}\text { High definition phase } \\
\text { contrast/fluorescence }\end{array}$ \\
\hline
\end{tabular}

Another approach for HTI uses laser-scanning fluorescence (LSF) cytometers that scans the entire depth of a well without Z-stack assembly or re-focusing [18]. Cellular resolution is achieved using scanning laser excitation coupled to photomultiplier detection (Supplementary Fig. S3). This configuration results in area-based scanning across a large field of view, plus simultaneous detection of up to four emission colors for efficient multiplexing. TTP Labtech's fourth generation LSF cytometers, Acumen® Cellista, allows users to combine biologically-relevant 3D cell culture models with high 
throughput methods, has been used to describe the formation of $3 \mathrm{D}$ cell cultures in a variety of formats [19-21]. Excitation beam penetrance is a challenge associated with image acquisition in 3D cell models. Imaging platforms that utilize multiphoton fluorescence technology, like two-photon excitation fluorescence [22], is an attractive alternative [23] because of its two-fold penetration depth compared with confocal microscopy [24]. While this technology is not currently suitable for HTI, it offers a glimpse into the future of 3D HTI systems.

An emerging technology called light sheet based fluorescence microscopy (LSFM, also known as selective or single plane illumination microscopy (SPIM)), which combines fluorescence optical sectioning with aspects of tomography, represents a promising approach for 3D imaging, especially with MCTS models [25]. LSFM exposes a specimen to up to 5000 times less energy than a confocal fluorescence microscopy, minimizing photo-bleaching and photo-induced cell damage. Light sheet illumination is becoming an important tool in 3D fluorescence microscopy, this technique holds great promise for the analysis of large numbers of samples with simple preparation, fast recording speed, high resolution and multi-channel fluorescence imaging [26]. LSFM imaging workstations have been used for dynamic long-term observations of living organoids [27, 28] and light sheet microscopy is commercially available from ZEISS, however, this technology has not yet been applied to HTI in 3D. The IncuCyte ZOOM High Definition Imaging system from Essen BioSciences greatly enhances phase-contrast imaging in microplates enabling applications such as live-cell non-perturbing imaging and can be used to track the formation, compactness, and uniformity of $3 \mathrm{D}$ culture 
models in real-time, all within the confines of a standard tissue culture incubator, allowing for long-term 3D culture analysis [29, 30]. The IncuCyte allows for cell based analysis in a variety of tissue culture plate formats including 96-384 well plates and provides 3D image analysis the form of maximal projections.

\subsection{Software}

With the technology to perform high-content image-based screens now accessible to many labs, there is an unmet need for tools that enable bench scientists to rapidly interpret large and complex image data sets. 3D HTI produces quantities of images that are well beyond the ability to be analyzed by manual inspection. The lack of applications to automatically reconstruct 3D models from 3D image stacks limits sophisticated optical methods from realizing their full potential. Recent advances in 3D imaging technology has inspired the establishment of new and rapidly growing bioimage open-source communities, more researchers are developing solutions to gain insights into the quantification of spheroids through computational analysis of large-scale image data sets. These software applications, such as Fiji/ImageJ [31], Vaa3D [32, 33], BioImageXD [34], TANGO [35] and Icy [36] (Table 2) are compatible with various computer operating systems (OS) and support many types of image file formats, including the standardized Open Microscopy Environment (OME) data model Bio-Formats [37]. 
Table 2. Available software designed for 3D image analysis.

\begin{tabular}{|c|c|c|c|}
\hline Software & Features & Availability & OS Platforms \\
\hline Fiji (ImageJ) & $3 \mathrm{D}$ viewer, $3 \mathrm{D}$ object counter & Open source & $\begin{array}{l}\text { Windows, Mac } \\
\text { and Linux }\end{array}$ \\
\hline Vaa3D & $\begin{array}{l}\text { Instant } 3 \mathrm{D} \text { visualization and annotation, } \\
\text { quantitative measurements }\end{array}$ & Open source & $\begin{array}{l}\text { Windows, Mac } \\
\text { and Linux }\end{array}$ \\
\hline BioImageXD & $\begin{array}{l}\text { Interactive 3D rendering tools with surface and } \\
\text { volume rendering algorithms }\end{array}$ & Open source & $\begin{array}{l}\text { Windows, Mac } \\
\text { and Linux }\end{array}$ \\
\hline Icy & $\begin{array}{l}\text { Intuitive visualization in 3D, specialized plug-ins } \\
\text { for } 3 \mathrm{D} \text { image processing and analysis }\end{array}$ & Open source & $\begin{array}{l}\text { Windows, Mac } \\
\text { and Linux }\end{array}$ \\
\hline TANGO & $\begin{array}{l}\text { Extract quantitative data from 3D fluorescence } \\
\text { imaging, integrated with } \mathrm{R} \text { for statistical analysis }\end{array}$ & Open source & $\begin{array}{l}\text { Windows, Mac } \\
\text { and Linux }\end{array}$ \\
\hline Volocity & $\begin{array}{l}\text { 3D rendering, deconvolution, volumetric } \\
\text { segmentation and measurement of 3D and 4D } \\
\text { image data }\end{array}$ & Commercial & $\begin{array}{l}\text { Windows, and } \\
\text { Mac }\end{array}$ \\
\hline Harmony & $\begin{array}{l}\text { Representing 3D positional information regarding } \\
\text { pixel maximal intensity into a 2D map for } \\
\text { visualization and automated analysis }\end{array}$ & Commercial & Windows \\
\hline $\begin{array}{l}\text { MetaMorph } \\
\text { (MetaXpress) }\end{array}$ & $\begin{array}{l}\text { Visualization of multi-dimensional data sets, 3D } \\
\text { isosurface viewing and rotation }\end{array}$ & Commercial & Windows \\
\hline Imaris & $\begin{array}{l}\text { Visualization, analysis, segmentation and } \\
\text { interpretation of } 3 \mathrm{D} \text { and } 4 \mathrm{D} \text { microscopy data sets }\end{array}$ & Commercial & $\begin{array}{l}\text { Windows, and } \\
\text { Mac }\end{array}$ \\
\hline Amira & $\begin{array}{l}\text { 3D data visualization, processing, and analysis, 3D } \\
\text { image segmentation and reconstruction }\end{array}$ & Commercial & $\begin{array}{l}\text { Windows, Mac } \\
\text { and Linux }\end{array}$ \\
\hline ImagePro & $\begin{array}{l}\text { 3D rendering and measurement, object counting, } \\
\text { creating animations of 3D Images }\end{array}$ & Commercial & Windows \\
\hline Huygens & $\begin{array}{l}\text { 3D image deconvolution, visualization and } \\
\text { analysis }\end{array}$ & Commercial & $\begin{array}{l}\text { Windows, Mac } \\
\text { and Linux }\end{array}$ \\
\hline
\end{tabular}

In addition to open source software, there are now a number of commercial software suites that are available for 3D visualization and complex analysis (Table 2). Many companies that develop and distribute 3D HTI/HCS hardware systems also offer tailor made 3D imaging software for optimized user experience and integrated approaches. For example, Volocity (PerkinElmer) and Metamorph (Molecular Devices) represent 3D analysis software that is integrated with their respective high-content imaging systems. Finally, there are third party companies developing 3D image processing software independent of the available high-content imaging systems, including: Imaris (Bitplane), 
Amira (FEI, Visualization Sciences Group), ImagePro (Media Cybernetics), Phaedra (Janssen Pharmaceutical) [38] and Huygens (Scientific Volume Imaging). In figure 1 we show, as examples, 3D-visualization using both Volocity and Vaa3D of the same data set consisting of a 3D liver microtissue section. This demonstration indicates that both software applications are capable of rendering multidimensional image data efficiently and precisely (Fig. 1). While commercial software applications offer more tools and features as well as support for 3D image analysis, open source software proves to be a reliable and cost effective alternative.

The available 3D image analysis software discussed in this review offer flexible and sophisticated toolboxes for 3D image segmentation and reconstruction. These software programs also contain features to measure shapes, volumes, and distances of the $3 \mathrm{D}$ objects with precision. However, all of these are low-throughput interactive applications that are not suitable for 3D HCS. Currently, Harmony (version 4.1), which drives PerkinElmer HCS instruments, can determine the axial position (Z-plane) of the brightest pixel at each $\mathrm{X}-\mathrm{Y}$ position in a volumetric image stack and represent this axial position information in a 2D map. This capability is demonstrated in figure 2 using the same sample data sets described in figure 1 . To the best of our knowledge, this is the only HTI platform that is capable of true 3D high-content analysis of thousands of microwells allowing for true HCS. 


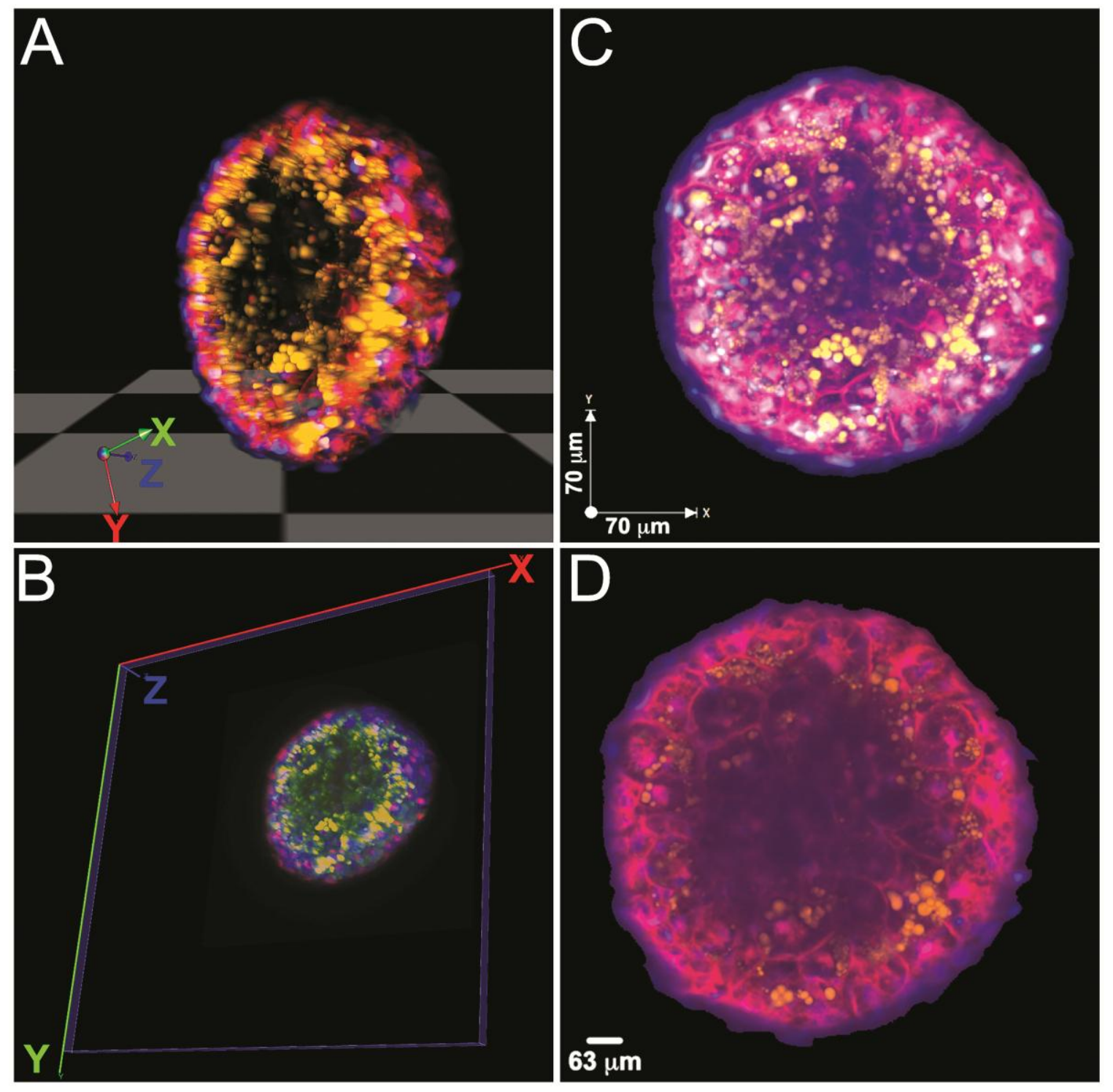

Fig. 1. Visualizing 3D image data using Volocity and Vaa3D. Liver cell spheroid stained with Hoechst 33342 (blue) to detect nuclei, CellMask DeepRed (red) to define individual cells and Nile Red (orange) to detect accumulation of lipids. (A) and (B), 3D view of the microtissue segment. (C) and (D) Maximal intensity projection of all 3 fluorescence channels is shown in overlay images. Microscopy data were captured using the 20x water immersion objective lens on an Opera Phenix. Image (A) and (C) were prepared using the Volocity (PerkinElmer); Image $(\mathbf{B})$ and $(\mathbf{D})$ were prepared using the Vaa3D (open source). 


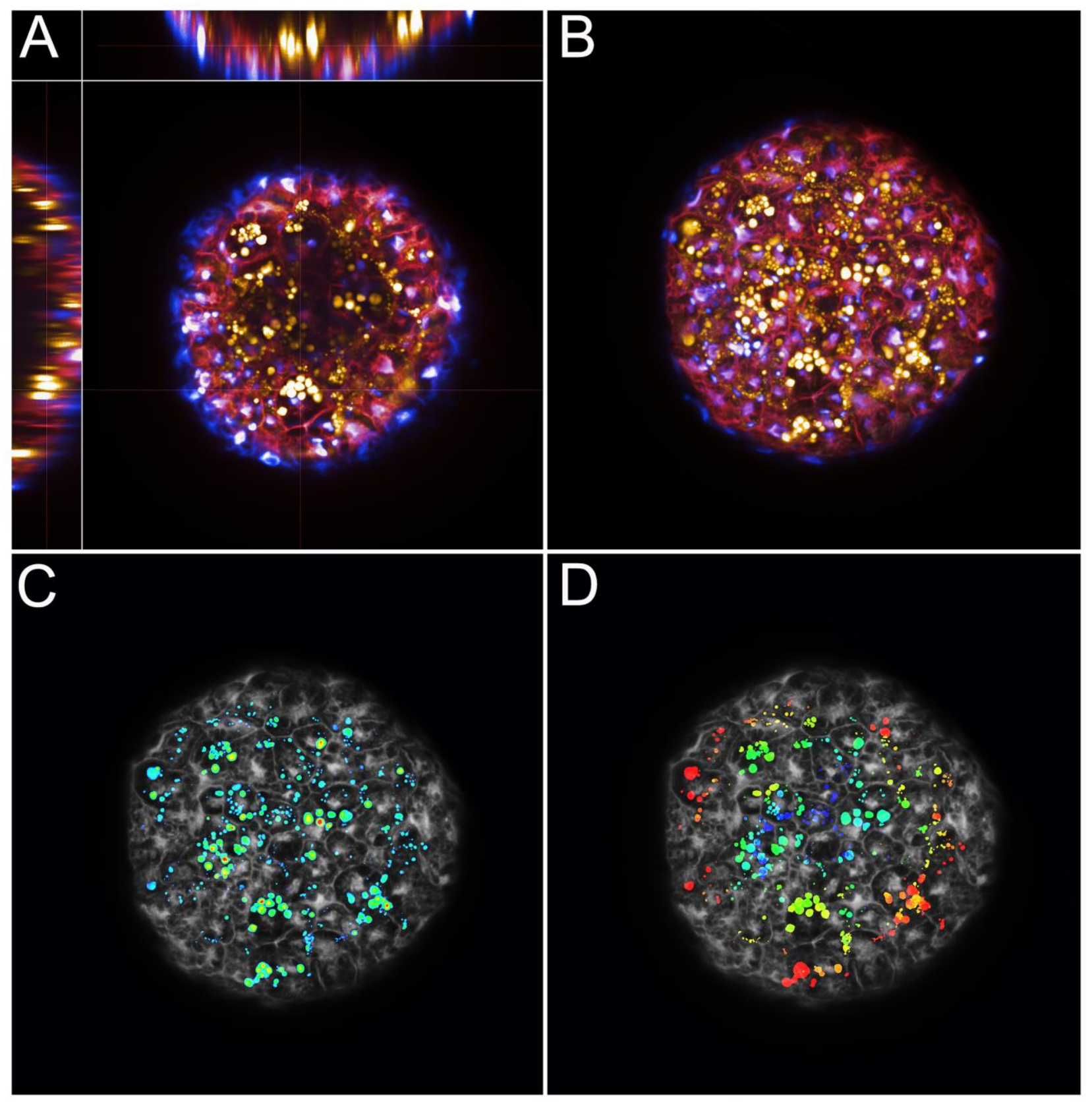

Fig. 2. Visualizing 3D image data using Harmony 4.1. Liver cell spheroid stained with Hoechst 33342 to detect nuclei, CellMask DeepRed to define individual cells and Nile Red to detect accumulation of lipids. (A) and (B), Multiple fluorescence channels are shown in overlay images. (A) Orthogonal views of the 3D data set, where the large image is a single focal plane. (B) Maximal intensity projection of all focal planes. (C) Pseudocolor represents signal intensity for the Nile Red channel in the maximal intensity projection. (D) Pseudocolor represents the axial position (Z-plane) of the brightest fluorescence intensity in the Nile Red channel of 3D image stack. Microscopy data were captured using the 20x water immersion objective lens on an Opera Phenix instrument. 
In summary, the transition of 3D HTI into a more quantitative technique will bring important scientific benefits in the form of new applications and improved performance and reproducibility. Visualization and quantification of MCTS and other 3D cell culture model can be accomplished faster and more economically by efficiently employing one or more of the versatile image analysis packages described herein.

\section{Progress towards 3D HTI and HCS drug discovery}

In recent years technical advances in imaging based technology has allowed for HTI/HCS based assays to be used in a number of 3D applications. In addition, various analysis protocols start to focus on deconstructing elements of cells cultured in 3D, such as morphological profiles and biomarker expression. For example, Wenzel and colleagues recently reported a HCS assay model of breast cancer using T47D MCTS uniformly arrayed in 384-well plates [39]. The model was used specifically to detect compounds that target dormant non-proliferating cells located within the spheroid interior. Following the screening of 1120 compounds, 9 respiratory chain inhibitors targeting these quiescent cells were identified, notably, diphenyleneiodonium. An interesting feature of this MCTS model was the use of spheroid permeable stains including Hoechst 33342 (nuclei stain) and SytoxGreen (DNA stain for dead cells in the interior of spheroids), eliminating the requirement of stable reporters. This 3D image acquisition

was accomplished using a custom built monolithic digital scanned laser light-sheet fluorescence microscope (mDSLM) $[40,41]$ and the 3D image reconstruction was done with Imaris software [39]. The significance of this study was that survival of these quiescent cells, displaying high drug resistance to conventional chemotherapy, were 
susceptible to cell death by blocking extracellular glucose transport to the interior cells of T47D MCTS. Astonishingly, this report showed that all 9 hits induced no cell death to T47D cells cultured in 2D but were potent inducers of cell death to dormant cells in $3 \mathrm{D}$ MCTS, demonstrating the superiority of 3D tissue culture models for novel drug discovery.

Similar to Wenzel et al., Reid and colleagues recently reported a valid HCS T47D MCTS model to track and suppress a subpopulation of quiescent breast CSCs marked by the biomarker cytokeratin 5 (CK5) [7]. CK5 is a marker of poor prognosis across all breast cancer and is expressed in basal-like triple negative breast cancer [42]. T47D cells are luminal breast cancer cells (estrogen receptor positive and progesterone receptor positive, $\mathrm{ER}^{+}$and $\mathrm{PR}^{+}$) that when exposed to progesterone induces the expansion of a subpopulation of $\mathrm{CSCs}$ that are both $\mathrm{ER}^{-}$and $\mathrm{PR}^{-}$, and $\mathrm{CK}^{+}$[43]. The $\mathrm{CK} 5^{+}$ subpopulation is enriched with stem and mesenchymal gene expression and are quiescent. This cell population is resistant to conventional chemotherapy and displays and invasive and metastatic phenotype [44]. T47D MCTS stably engineered with a CK5 promoter green fluorescent protein (CK5Pro-GFP) reporter was used to track the expansion of the CSC subpopulation after progesterone treatment. Using this model Reid et al. observed a 35-fold expansion of the CSC phenotype and after screening approximately 300 compounds from the NIH clinical collection they identified 4 hits, including 3 retinoids (e.g. retinoic acid) that were potent suppressors of the CSC phenotype expansion $\left(\mathrm{IC}_{50}=\right.$ $3 \mathrm{nM})$. This 3D HCS utilized the Operetta high-content imaging system from PerkinElmer equipped with Harmony software to develop an image analysis algorithm of CK5Pro-GFP expression in MCTS that was used to capture data in a single Z-stack plane 
within a MCTS and globally by using maximal projections [42].

Recently, Kenny and colleagues developed and implemented an ovarian cancer 3D organotypic model mimicking the omentum and peritoneum histology by layering primary human fibroblasts, mesothelial cells, and collagen I ECM with either SKOV3iplGFP or HeyA8-GFP ovarian cancer cells forming the top layer [45]. This model was designed to identify small molecules that were effective at inhibiting ovarian cancer cell adhesion, migration and invasion. The migratory fluorescent ovarian cancer cells in the assay were analyzed using a TTP Labtech Acumen eX3 fluorescent cytometer. The primary HCS model was formatted in both 384-well and 1536-well plates, and miniaturization of the assay into 1536-well plates did not compromise assay quality. Approximately, 1,000 compounds were screened with this HCS model resulting in 15 active hits, notably, beta-escin, which displayed potent inhibition of adhesion, migration, and invasion in vitro and was confirmed using in vivo models of metastasis. The authors concluded that organotypic models for human disease are practical and useful for rapidly identifying effective drugs with a lower attrition rate compared to simpler HTS drug discovery.

An interesting study by Krausz et al. described an anchorage-dependent 3D co-culture model including tumor cells and stromal cells organized in a complex ECM microenvironment [46]. This model was used to assess tumor growth and focused on non-uniform 3D-colony structures as opposed to uniform single MCTS. The model was validated for HCS in 96-384 well plates using PC-3M prostate cancer cells, achieving a throughput capability of 10,000 compounds per week. Interestingly, the authors compared various imaging platforms (MIAS-2, IN Cell Analyzer 2000, and Opera) and 
their respective analysis programs along with their in house tool, Plate-based HighContent Analysis Evaluation and Dynamic Reliability Assurance (Phaedra). The results of the comparison indicated that all of the platforms and image analysis software provided very similar results and that none of the imaging systems were superior.

Although organoid/MCTS models of cancer have been the most widely used 3D culture models for HTI/HCS drug discovery, other 3D models are on the verge of mainstream use such as organoid culture models for toxicology studies [4]. Drug-induced hepatotoxicity is the main cause for drug attrition resulting in 30-50\% liver failure observed during clinical trials [47]. Thus, there is a need for more predicative in vitro models of liver toxicology to identify liver toxicophores during early drug development. To this end, Gunness and colleagues have recently reported a 3D HepaRG liver cell spheroid model useful to identify drug-induced hepatotoxicity [48]. The authors concluded that the $3 \mathrm{D}$ model was superior to the mainstay $2 \mathrm{D}$ cell models used to assess acute drug induced liver toxicity and would likely be useful for assessing chronic liver toxicity due to the available long-term culture capability with 3D organoids. While the organoid model reported by Gunness et al. was not developed for HTI, models adapted for HTI toxicity screening may benefit greatly from the spatiotemporal information gained from image based analysis. Qin Meng discusses the use of liver organoids for HCS toxicology in a recent expert opinion review [49].

\section{Conclusions and outlook}

While 3D organotypic culture models have existed for a century, advances in miniaturized organoid culture methods, high-throughput imaging systems (hardware and software), chemical and biological probes, and gene reporter readouts are converging to 
make automated HTI/HCS analysis practical. Thus, we are entering a renaissance period of organotypic culture models driven by the current technology allowing for HTI/HCS drug discovery and toxicological screening. This review has focused on the state-of-theart HTI technology and recent literature examples of 3D organotypic culture models employing this technology for drug discovery toxicology screening, as well as a few organoid models that are on the verge of utilizing HTI/HCS capability. The application of these 3D models has demonstrated superiority over 2D-cell culture models in identifying novel hits and leads and their respective mechanism(s) of action or organ toxicity. Importantly, these discoveries were done using $\leq 1,000$ compounds, indicating their potential for discovery once the HTI throughput can accommodate large and diverse chemical libraries (thousands to millions) or even focused chemical libraries with superior chemical scaffolds (e.g. natural products). Nonetheless, there is still a significant amount of research required to establish 3D organotypic culture models that not only possess the essential elements of the human organ or disease but also are automated and analyzed in a high-throughput manner. To date, 3D tumor models have been more widely adapted for use with HTI/HCS, which is likely due to the ease in culture and self assembly compared to other non-cancer induced pluripotent stem cells (iPSC) derived organoid models. However, these organoid models show a promising future as an outstanding platform for systems biology HTI/HCS drug discovery and chemical toxicological screening $[3,4,50-53]$. As 3D HTI technology continues to advance 2Dcell based assays will be phased out leaving organotypic culture models as the main discovery pipeline, yielding more effective and less toxic drugs therapies for the treatment of human disease. 


\section{Acknowledgements}

Dr. LaBarbera's research is currently supported by the NIH National Eye Institute under award number R01EY021498 and a career development award from the Department of Defense [Peer Reviewed Cancer Research Program] under award number W81XWH-131-0344. The authors thank the High-throughput screening and chemical biology core facility High at the University of Colorado AMC Skaggs School of Pharmacy and Pharmaceutical Sciences for contributions in 3D software data analysis.

\section{References}

[1] M. Srivastava, O. Simakov, J. Chapman, B. Fahey, et al., Nature, 466 (2010) 720726.

[2] H.V. Wilson, Science, 25 (1907) 912-915.

[3] M.A. Lancaster, J.A. Knoblich, Science, 345 (2014) 1247125.

[4] A. Ranga, N. Gjorevski, M.P. Lutolf, Advanced drug delivery reviews, 69-70 (2014) 19-28.

[5] R.M. Sutherland, J.A. McCredie, W.R. Inch, J Natl Cancer Inst, 46 (1971) 113120.

[6] D.V. LaBarbera, B.G. Reid, B.H. Yoo, Expert Opin Drug Dis, 7 (2012) 819-830.

[7] B.G. Reid, T. Jerjian, P. Patel, Q. Zhou, et al., Curr Chem Genom Transl Med, 8 (2014) 27-35.

[8] A. Amann, M. Zwierzina, G. Gamerith, M. Bitsche, et al., PLoS One, 9 (2014) e92511.

[9] J. Friedrich, R. Ebner, L.A. Kunz-Schughart, Int. J. Radiat. Biol., 83 (2007) 849871.

[10] R.Z. Lin, H.Y. Chang, Biotechnology J., 3 (2008) 1172-1184.

[11] A. Abbott, Nature, 424 (2003) 870-872.

[12] S. Ghosh, G.C. Spagnoli, I. Martin, S. Ploegert, et al., J. Cell. Physiol., 204 (2005) $522-531$.

[13] J. Han, H. Chang, O. Giricz, G.Y. Lee, et al., PLoS Comput. Biol., 6 (2010) e1000684.

[14] K. Borgmann, O. Zschenker, T. Streichert, S. Hehlgans, et al., PLoS One, 7 (2012) e34279.

[15] J.G. Moffat, J. Rudolph, D. Bailey, Nat. Rev. Drug. Discov., 13 (2014) 588-602.

[16] D.C. Swinney, J. Anthony, Nat. Rev. Drug. Discov., 10 (2011) 507-519.

[17] M.E. Dickinson, Dev. Dyn., 235 (2006) 2386-2400.

[18] W.P. Bowen, P.G. Wylie, Assay Drug Dev. Technol., 4 (2006) 209-221.

[19] S.R. Horman, J. To, A.P. Orth, J. Biomol. Screen., 18 (2013) 1298-1308.

[20] S.R. Horman, J. To, A.P. Orth, N. Slawny, et al., Nat. Methods, 10 (2013). 
[21] P.G. Wylie, D.J. Onley, A.F. Hammerstein, W.P. Bowen, Assay Drug Dev. Technol., 13 (2015) 66-78.

[22] R.K. Benninger, D.W. Piston, Curr. Protoc. Cell Biol., 59 (2013) 4.11.1-4.11.24.

[23] W. Göbel, B.M. Kampa, F. Helmchen, Nat. Methods, 4 (2007) 73-79.

[24] R.J. Gilbert, M. Hoffman, A. Capitano, P.T.C. So, Microsc. Res. Tech., 51 (2000) 204-210.

[25] F. Pampaloni, N. Ansari, E.H.K. Stelzer, Cell Tissue Res., 352 (2013) 161-177.

[26] F. Pampaloni, E.H.K. Stelzer, A. Masotti, Recent Pat. Biotechnol., 3 (2009) 103-

117.

[27] F. Pampaloni, R. Richa, N. Ansari, E.H.K. Stelzer, Methods Mol. Biol., 1251 (2015) 43-57.

[28] F. Pampaloni, U. Berge, A. Marmaras, P. Horvath, et al., Integr. Biol., 6 (2014) 988-998.

[29] O. Aftab, M. Fryknäs, U. Hammerling, R. Larsson, et al., J. Biomol. Screen., 20 (2015) 372-381.

[30] V. Härmä, H.P. Schukov, A. Happonen, I. Ahonen, et al., PLoS One, 9 (2014) e96426.

[31] J. Schindelin, I. Arganda-Carreras, E. Frise, V. Kaynig, et al., Nat. Methods, 9 (2012) 676-682.

[32] H. Peng, A. Bria, Z. Zhou, G. Iannello, et al., Nat. Protoc., 9 (2014) 193-208.

[33] H. Peng, J. Tang, H. Xiao, A. Bria, et al., Nat. Commun., 5 (2014) 4342.

[34] P. Kankaanpää, L. Paavolainen, S. Tiitta, M. Karjalainen, et al., Nat. Methods, 9 (2012) 683-689.

[35] J. Ollion, J. Cochennec, F. Loll, C. Escudé, et al., Bioinformatics, 29 (2013) 1840-1841.

[36] F. de Chaumont, S. Dallongeville, N. Chenouard, N. Hervé, et al., Nat. Methods, 9 (2012) 690-696.

[37] M. Linkert, C.T. Rueden, C. Allan, J.M. Burel, et al., J. Cell Biol., 189 (2010) 777-782.

[38] F. Cornelissen, M. Cik, E. Gustin, J. Biomol. Screen., 17 (2012) 496-506.

[39] C. Wenzel, B. Riefke, S. Gründemann, A. Krebs, et al., Exp. Cell Res., 323 (2014) 131-143.

[40] P.J. Verveer, J. Swoger, F. Pampaloni, K. Greger, et al., Nat. Methods, 4 (2007) 311-313.

[41] P.J. Keller, A.D. Schmidt, A. Santella, K. Khairy, et al., Nat. Methods, 7 (2010) 637-642.

[42] D.M. Abd El-Rehim, S.E. Pinder, C.E. Paish, J. Bell, et al., J. Pathol., 203 (2004) 661-671.

[43] S.D. Axlund, B.H. Yoo, R.B. Rosen, J. Schaack, et al., Hormones \& cancer, 4 (2013) 36-49.

[44] P. Kabos, J.M. Haughian, X. Wang, W.W. Dye, et al., Breast Cancer Res Tr, 128 (2011) 45-55.

[45] H.A. Kenny, M. Lal-Nag, E.A. White, M. Shen, et al., Nat. Commun., 6 (2015) 6220 .

[46] E. Krausz, R. de Hoogt, E. Gustin, F. Cornelissen, et al., J Biomol Screen, 18 (2013) 54-66. 
[47] G. Tuschl, B. Lauer, S.O. Mueller, Expert opinion on drug metabolism \& toxicology, 4 (2008) 855-870.

[48] P. Gunness, D. Mueller, V. Shevchenko, E. Heinzle, et al., Toxicol. Sci., 133 (2013) 67-78.

[49] Q. Meng, Expert opinion on drug metabolism \& toxicology, 6 (2010) 733-746.

[50] M. Huch, S.F. Boj, H. Clevers, Regen. Med., 8 (2013) 385-387.

[51] M. Huch, C. Dorrell, S.F. Boj, J.H. van Es, et al., Nature, 494 (2013) 247-250.

[52] M. van de Wetering, H.E. Francies, J.M. Francis, G. Bounova, et al., Cell, 161 (2015) 933-945.

[53] M.A. Lancaster, J.A. Knoblich, Nat. Protoc., 9 (2014) 2329-2340. 